\title{
Primary Small Cell Breast Carcinoma-A Rare Diagnosis: Management Strategies and a Brief Literature Review
}

ISSN: 2578-0379

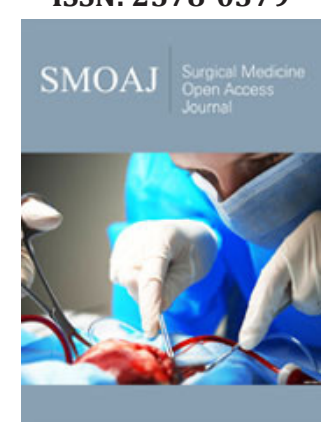

*Corresponding author: Jameel Ali, Department of Surgery, Trinidad and Tobago

Submission: 海 September 16, 2019

Published: 眥September 24,2019

Volume 3 - Issue 1

How to cite this article: Ali J, Spence $\mathrm{R}$, Milne D, Jarvis J, Olivier L, Barrow M, Sookar N. Primary Small Cell Breast Carcinoma-A Rare Diagnosis: Management Strategies and a Brief Literature Review. Surg Med Open Acc J.3(1). SMOAJ.000553.2019. DOI: 10.31031/SMOAJ.2019.03.000553.

Copyright@ Jameel Ali, This article is distributed under the terms of the Creative Commons Attribution 4.0 International License, which permits unrestricted use and redistribution provided that the original author and source are credited.
Jameel Ali, David Milne, Lyronne Olivier, Richard Spence, Navin Sookar, Johnathan Jarvis and Mikhail Barrow

Department of Surgery, Trinidad and Tobago

\begin{abstract}
Primary small cell breast carcinoma represents less than $1 \%$ of breast cancers. Due to its rarity, there are no uniformly accepted guidelines for treatment. Its prognosis is varied being generally regarded as worse than that of most breast cancers and it poses unique diagnostic challenges. We present a case of primary small cell breast cancer, rationale for our management strategies with reference to the published literature to serve as a guide to the management of this rare cancer of the breast.
\end{abstract}

Keywords: Neuroendocrine breast cancer; Small cell breast carcinoma; Treatment of small cell breast carcinoma

\section{Introduction}

Neuroendocrine Breast Cancers (NEBC) are reported in approximately 1\% of all breast cancers with the small cell variety being the rarest and most poorly differentiated subtype [1]. Although neuro endocrine malignant tumors are frequently of pulmonary origin [2], extrapulmonary sites of this malignancy are reported in most other organs such as the GI tract, trachea ,larynx, bladder, prostate, cervix, ovaries [2]. In the breast, although the exact histogenesis of these tumors is unknown, they are thought to arise from aberrant differentiation of a neoplastic stem cell or less likely derived from neural crest cells that migrate to the breast or originate in neuroendocrine cells of the breast [3] .There are many conflicting reports on the prognosis of NEBC, some reports suggesting that they are less aggressive than the usual invasive ductal carcinoma [4] while others suggest a poorer prognosis [3]. As in invasive ductal carcinoma, high grade, larger tumor size, the presence of lymph node and distant metastasis are associated with poorer prognosis. The small cell subtype of NEBC is however generally associated with a poorer prognosis [5]. As described in our case presentation to follow, specific criteria must be met before a diagnosis of primary small cell breast carcinoma is made, first of all to exclude a lung or other primary source and to confirm that the immunohistochemical features are in keeping with a breast primary.

\section{Case History}

Mrs KB is a 37 year old premenopausal previously healthy mother of 3 breast fed healthy children. She presented to our facility in July 2018 with a 6 month history of an enlarging tender clinically hard, solid irregular but mobile right breast mass. Ultrasound and mammography demonstrated a $6.6 \mathrm{~cm}$ irregular mass with architectural distortion, with no muscle or chest wall involvement. Bilateral benign appearing axillary nodes were noted and a $1.7 \mathrm{~cm}$ benign appearing mass in the left breast. Trucut biopsy of the left breast mass showed fibro adenomatous changes with no evidence of atypia or malignant cells but trucut biopsy of the right breast mass showed small to medium sized hyperchromatic cells with frequent mitotic figures, focal necrosis with scattered large pleomorphic cells consistent with a high grade small cell carcinoma see Figure 1. The cells tested positive for Chromogranin and Synaptophysin and were triple negative (Estrogen receptor, Progesterone receptor and Her2 Neu2 negative). Further immune markers using specific stains to rule out a thoracic or pulmonary origin of the cells TTf1 see Figure 2 and to confirm the mammary origin of the cells of the tumor GATA-3 see Figure 3 were used to confirm that the cells of the tumor were primarily breast in origin. 


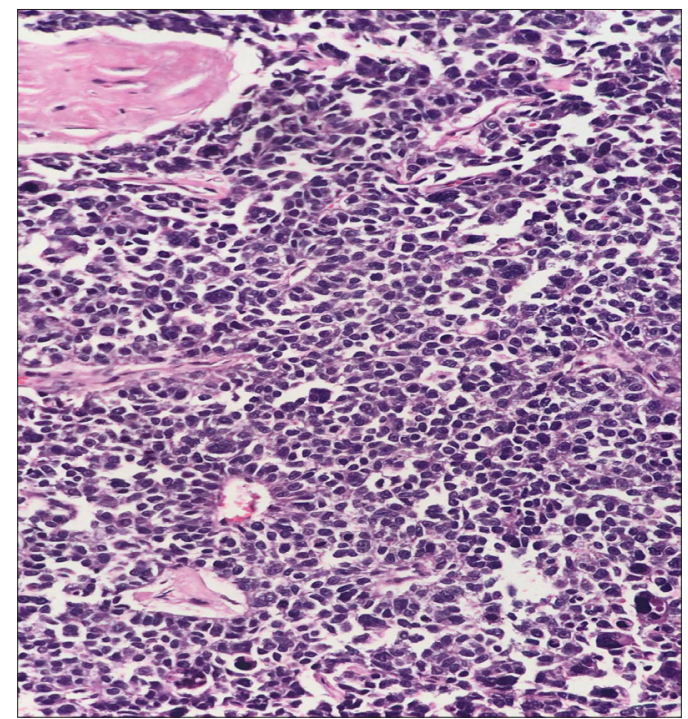

Figure 1: Trucut biopsy of the right breast mass showed small to medium sized hyperchromatic cells with frequent mitotic figures, focal necrosis with scattered large pleomorphic cells consistent with a high grade small cell carcinoma.

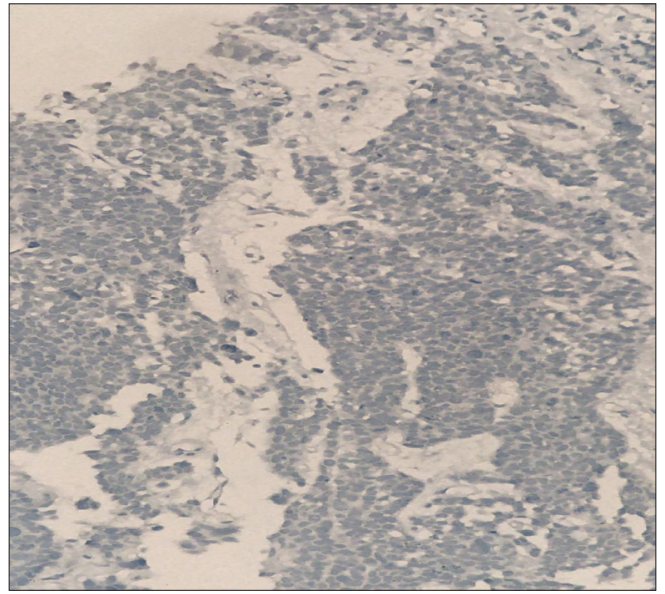

Figure 2: Immune markers using specific stains ruled out a thoracic or pulmonary origin of the cells (TTF1 negative).

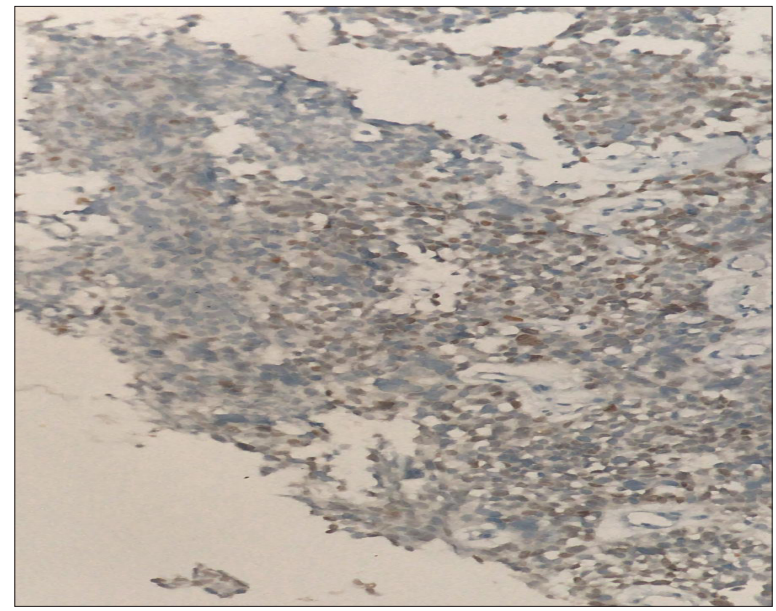

Figure 3: To confirm the mammary origin of the cells of the tumor GATA-3 were used to confirm that the cells of the tumor were primarily breast in origin. 
Preoperative CT scan of the Chest, Abdomen and Pelvis showed no evidence of metastatic disease in the lungs, liver, bone or other lymphatic beds in the chest or abdomen and further confirming the absence of a primary small cell carcinoma of the lung. A separate CT scan of the head also showed no evidence of an intracranial lesion. At our Multidisciplinary Tumor Board Rounds, the consensus was to proceed to neoadjuvant chemotherapy starting with Cisplatin and Taxotere. After 2 cycles of this chemotherapy regime there was a dramatic decrease in the size of the tumor from $6.6 \mathrm{~cm}$ to $1.7 \mathrm{~cm}$ and the decision was made to proceed to mastectomy and Axillary clearance with the plan to proceed to adjuvant therapy after surgery followed by a full 5 weeks course of external chest wall radiation. The pathology report post mastectomy described the same types of cells as in the biopsy with clear margins and all 10 recovered axillary nodes tested negative for metastasis and the repeat routine Immunohistochemistry stains showed triple negative disease.

She has completed the full course of chemotherapy and radiotherapy. A one year follow up CT scan of the chest abdomen and pelvis done in August 2019 again showed no evidence of metastatic or mammary cancer. The patient continues to maintain her weight, with good appetite and no clinical evidence of cancer.

\section{Discussion}

Recognizing the very low frequency of the diagnosis in our described patient we sought available information in the literature to guide our management strategy. Not surprisingly, there was a lack of consensus from our review, particularly in the realm of specific therapy for this form of breast cancer because most of the reported literature are on case studies with no large randomized clinical trials. The literature was very helpful in emphasizing the importance of making the correct diagnosis. We are confident that we have made the correct diagnosis by obtaining a reliable tissue sample in the form of a core needle biopsy and to have the specimen examined thoroughly by at least 2 very experienced competent pathologists who identified the role of specific immunochemistry markers-positive for synaptophysin and chromogranin as well as negative TTf1 marker to rule out a lung source and positive GATA-3 markers confirming breast as the primary source of the cancer [3].

Another feature recommended in the diagnostic work up is aimed at excluding another primary source of NEBC [6]. In our case, CT scan of chest abdomen and pelvis, mammogram and ultrasound as well as Head CT showed no other source for a primary or secondary site of the breast tumor. Additional imaging such as PET scan and MRI are reported in some of the case reports but we did not think this would add to the clarity of the diagnosis, especially in a low income environment such as ours where cost could be a factor in managing our patients through additional expensive imaging.

In searching the literature for guidance on therapy specific for primary small cell carcinoma of the breast, there is no consensus on the role and type of surgery, neoadjuvant and adjuvant chemotherapy or radiotherapy because most of the literature consists of isolated case studies. The largest population based series by Hare et al involved 199 cases comparing small cell breast carcinoma with small cell carcinoma of the lung [7].

\section{The role and type of chemotherapy}

Although neoadjuvant chemotherapy is being used increasingly for T1 and T2 tumors of the breast [8] this form of therapy is still used traditionally for locally advanced (T3 and larger) lesions. In our case, the patient met criteria for locally advanced breast carcinoma so the decision to proceed to neoadjuvant therapy was justified. There is no consensus on the type of chemotherapy to use. Regimens for invasive ductal carcinoma are applied to NEBC by many [9] including anthracycline based therapy with varying results [8]. Cisplatin based chemotherapy as in small cell carcinoma of the lung is also recommended by many [10]. In our patient Cisplatin with Taxotere was used after discussion among our medical oncologists. Our decision was made easier when the patient had such a dramatic positive response. If the response was not positive we were prepared to pursue other alternative regimens after further discussions among our group. Because of the prohibitive cost of utilizing genomic panel technology for predicting likelihood of response to chemotherapy we did not pursue this option for selecting the type of chemotherapy for our patient.

\section{The role and type of surgery}

As with chemotherapy, the role of breast conservation surgery versus mastectomy is controversial. Increasingly, surgeons are using breast conservation surgery after downsizing of tumor with neoadjuvant therapy [11]. Despite the dramatic decrease in size with neoadjuvant therapy we discussed the surgical options with the patient but recommended mastectomy which she accepted because of the lack of data on breast conservation surgery for locally advanced small cell breast carcinoma. We also discussed the role of breast reconstruction but decided that this will be revisited after at least 2 years of disease free survival.

\section{The role of endocrine therapy}

Many of these tumors are estrogen receptor positive and most are Her2Neu2 negative [1] so in ER positive tumors, antiestrogen therapy is frequently applied as in regular invasive ductal carcinoma. Our patient had triple negative disease so no endocrine therapy was recommended.

\section{Conclusion}

Primary small cell breast carcinoma is rare with very limited literature to guide management. We have presented a case history to explore the decision making process in this cancer, giving our rationale for our therapeutic decisions of prime importance is differentiating primary small cell carcinoma from other pulmonary or extrapulmonary sources utilizing expertise in pathologic assessment including immunohistochemistry and biomarker profiles as well as imaging modalities. There seems to be a role for neoadjuvant chemotherapy at least for $\mathrm{T} 2$ and larger tumors . There also seems to be a role for breast conservation surgery but patients should be informed about the lack of consensus in this very rare 
disease and unless the patient feels strongly otherwise, we would recommend mastectomy with axillary node sampling.

The type of chemotherapy is also controversial including neoadjuvant chemotherapy. We would recommend neoadjuvant therapy for locally advanced disease and reserve neoadjuvant therapy for early disease on an individualized basis after consulting with colleagues and the patient because of lack of clarity in the literature. Except for triple negative disease as our patient had, it seems appropriate to recommend antiestrogen therapy in estrogen positive tumors as in invasive ductal carcinoma. Finally, although this is not stated clearly in the literature we will consider offering all modalities of treatment i.e. surgery, radiation and chemotherapy in all these patients because of the lack of consensus arising from the small numbers of cases and the prohibitive cost of utilizing genomic profiling to more precisely predict response to specific chemotherapy. Whereas all therapeutic modalities have their risks, these are usually not life threatening if applied with caution, whereas omission of a possibly beneficial therapeutic modality because of lack of supporting data could adversely affect outcome.

\section{References}

1. Dalle IA, Abbas J, Boulos F, Salem Z, Assi HI (2017) Primary small cell carcinoma of the breast: A case report. Journal of Medical Case Reports 11(1): 290

2. Yao JC, Hassan M, Phan A, Dagohoy C, Leary C, et al. (2008) One hundred years after carcinoid: Epidemiology of and prognostic factors for neuroendocrine tumors in 35,825 cases in the United States. Journal of Clinical Oncology 26(18): 3063-3072.
3. Rosen LE, Gattuso P (2017) Neuroendocrine tumors of the breast Archives of Pathology \& Laboratory Medicine 141(11): 1577-1581.

4. López BE, Alonso RM, Barraza G, Vazquez MA, Bernadó L, et al. (2008) Solid neuroendocrine breast carcinomas: Incidence, clinico-pathological features and immunohistochemical profiling. Oncology Reports 20(6): 1369-1374.

5. Tian Z, Wei B, Tang F, Wei W, Gilcrease MZ, et al. (2011) Prognostic significance of tumor grading and staging in mammary carcinomas with neuroendocrine differentiation. Human Pathology 42(8): 1169-1177.

6. Adams RW, Dyson P, Barthelmes L (2014) Neuroendocrine breast tumors: Breast cancer or neuroendocrine cancer presenting in the breast? Breast 23(2): 120-127.

7. Hare F, Giri S, Patel JK, Hahn A, Martin MG (2015) A population-based analysis of outcomes for small cell carcinoma of the breast by tumor stage and the use of radiation therapy. Springerplus 4(1): 138.

8. Simmons CE (2018) The changing role of neoadjuvant therapy in breast cancer, considering systemic treatment for patients with operable as well as inoperable disease. BCMJ 60(2): 103-108.

9. Inno A, Bogina G, Turaza M, Bortesi L, Duranti S, et al. (2016) Neuroendocrine carcinoma of the breast: Current evidence and future perspectives. The Oncologist 21(1): 28-32.

10. Karim SM, Zekri J (2012) Chemotherapy for small cell lung cancer: A comprehensive review. Oncology Reviews 6(4): 1-36.

11. Arlow RL, Paddock LE, Niu X, Kirstein L, Haffty BG, et al. (2018) Breast conservation therapy after neoadjuvant chemotherapy does not compromise 10-year breast cancer-specific mortality. Am J Clin Oncol 41(12): 1246-1251.

For possible submissions Click below: 